\title{
On branches at infinity of a pencil of polynomials in two complex variables
}

\author{
by T. KRASIŃSKI (Łódź)
}

\begin{abstract}
Let $F \in \mathbb{C}[x, y]$. Some theorems on the dependence of branches at infinity of the pencil of polynomials $f(x, y)-\lambda, \lambda \in \mathbb{C}$, on the parameter $\lambda$ are given.

1. Introduction. W. Engel [2] took the following fact for granted: "For a special member of the pencil $f(x, y)-\lambda, \lambda \in \mathbb{C}$, the number of branches at infinity cannot be greater than the corresponding number for the general one" and used it in a proof of Keller's Jacobian Conjecture. T. T. Moh [3] claims the falsehood of the above statement, quoting a counterexample (unpublished) given to him by S. S. Abhyankar. T. T. Moh proves there that if $f(x, y)$ has only one branch at infinity, then so does each element of the pencil $f(x, y)-\lambda, \lambda \in \mathbb{C}$.

We obtain some results on branches of the pencil of polynomials $f(x, y)-$ $\lambda, \lambda \in \mathbb{C}$, without any additional assumptions. Namely, we prove that the number of branches at infinity of polynomials of this pencil is constant in the plane of the variable $\lambda$, excluding a finite set which is effectively defined (see Theorem 1). Moreover, outside these "bad" points, the branches at infinity have parametrizations analytically depending on $\lambda$ (see Theorem 2).

In the last section we give examples which show that the above number of branches at infinity (even counted with multiplicities) is neither lower nor upper semicontinuous. The first of these examples disproves Engel's statement. Both examples contradict a proposition of S. S. Abhyankar given by T. T. Moh [3].
\end{abstract}

2. Branches at infinity. For every $R>0$ and $t_{0} \in \mathbb{C}$, we put $K\left(t_{0}, R\right)=\left\{t \in \mathbb{C}:\left|t-t_{0}\right|<R\right\}, K(R)=\{t \in \mathbb{C}:|t|>R\}$. Further, for an open set $U \subset \mathbb{C}^{n}$, we denote by $\mathcal{O}(U)$ the ring of holomorphic functions in $U$. We shall consider the space $\mathbb{C}^{2}$ as being imbedded in the complex projective

1991 Mathematics Subject Classification: 32A99, 14H05. 
space $\mathbb{P}^{2}$ in the following way: $\mathbb{C}^{2} \ni(x, y) \mapsto(x: y: 1) \in \mathbb{P}^{2}$. Denote by $U_{1}$, $U_{2}, U_{3}$ the canonical affine subspaces of $\mathbb{P}^{2}$, i.e. $U_{1}=\left\{(x: y: z) \in \mathbb{P}^{2}: x \neq 0\right\}$ and similarly for $U_{2}, U_{3} ; \varrho_{1}, \varrho_{2}, \varrho_{3}$ are the canonical maps of these subspaces onto $\mathbb{C}^{2}$, i.e. $\varrho_{1}: U_{1} \rightarrow \mathbb{C}^{2}, \varrho_{1}((x: y: z))=(y / x, z / x)$ and similarly for $\varrho_{2}, \varrho_{3}$.

Let $F \in \mathbb{C}[x, y], f \neq$ const. Let $V$ be the set of zeros of $f$ in $\mathbb{C}^{2}$ and $\bar{V}$ its closure in $\mathbb{P}^{2}$. As is known, $\bar{V}$ is the set of zeros in $\mathbb{P}^{2}$ of the homogenization $f^{*}$ of $f$. Let $f_{1}, f_{2} . f_{3}$ denote the canonical holomorphic functions generated by $f^{*}$ in $U_{1}, U_{2}, U_{3}$, respectively, i.e. $f_{1}: U_{1} \rightarrow \mathbb{C}$, $f_{1}((x: y: z))=f^{*}(1, y / x, z / x)$ and similarly for $f_{2}$ and $f_{3}$.

Let $P_{1}, \ldots, P_{k}$ be the points of $f$ at infinity, i.e. the common points of $\bar{V}$ and the line at infinity $H_{\infty}=\{(x: y: z): z=0\}$. Take one of these points, say $P_{i}$. There exists $j$ such that $P_{i} \in U_{j}$. Consider the germ $\left(f_{j}\right)_{P_{i}}$ of $f_{j}$ in the ring $\mathcal{O}_{P_{i}}$ of germs of holomorphic functions at $P_{i}$. Let $\left(f_{j}\right)_{P_{i}}=\xi_{1}^{l_{1}} \ldots \xi_{m}^{l_{m}}$ be a factorization into irreducible and non-associated factors in $\mathcal{O}_{P_{i}}$. Then the germ $\bar{V}_{P_{i}}$ of the set $\bar{V}$ at $P_{i}$ has a decomposition $\bar{V}_{P_{i}}=V\left(\xi_{1}\right) \cup \ldots \cup V\left(\xi_{m}\right)$ into the union of irreducible germs. Obviously, the germs $V\left(\xi_{s}\right)$ and the exponents $l_{s}$ do not depend on the choice of the set $U_{j}$ which contains $P_{i}$ because in each intersection $U_{j_{1}} \cap U_{j_{2}}, j_{1} \neq j_{2}$, the holomorphic functions $f_{j_{1}}$ and $f_{j_{2}}$ differ by a holomorphic nowhere vanishing factor. Thus the following definition makes sense.

Definition. Each of the germs $V\left(\xi_{s}\right), s=1, \ldots, m$, is called a branch of $f$ at infinity at $P_{i}$ (or shortly, a branch of $f$ at $P_{i}$ ), whereas the exponent $l_{s}$ of the factor $\xi_{s}$ in the factorization of $\left(f_{j}\right)_{P_{i}}$ is called the multiplicity of the branch $V\left(\xi_{s}\right)$. The number of branches of $f$ at $P_{i}$ is denoted by $r_{P_{i}}(f)$, and when counted with multiplicities, by $\widetilde{r}_{P_{i}}(f)$. The set of branches of $f$ at infinity at all points $P_{i}, i=1, \ldots, k$, is called the set of branches of $f$ at infinity, their number being denoted by $r(f)$, and when counted with multiplicities, by $\widetilde{r}(f)$.

Note that the number of branches of $f$ at infinity and their multiplicities do not depend on the choice of a linear coordinate system in $\mathbb{C}^{2}$ because any linear change of coordinates in $\mathbb{C}^{2}$ extends to a biholomorphism of $\mathbb{P}^{2}$ preserving the line at infinity. So, in the sequel, we shall assume that the polynomial $f$ has the form

$$
\begin{aligned}
& f(x, y)=y^{n}+a_{1}(x) y^{n-1}+\ldots+a_{n}(x), \\
& \quad a_{i} \in \mathbb{C}[x], \operatorname{deg} a_{i} \leq i, i=1, \ldots, n, n \geq 1 .
\end{aligned}
$$

This implies that the points of $f$ at infinity lie in $U_{1}$, and that $\operatorname{deg} f=n$. Moreover, if $f$ has the form (1), then the branches of $f$ at infinity and their multiplicities can be characterized in the ring $\mathcal{M}[y]$ where $\mathcal{M}$ is the field of germs of meromorphic functions in $x$ at the point $\infty \in \overline{\mathbb{C}}$. Namely, we have the following more or less known 
Proposition. Let $\widehat{f}$ be the element in $\mathcal{M}[y]$ generated by a polynomial $f$ of the form (1). Let $\widehat{f}=\widehat{F}_{1}^{l_{1}} \ldots \widehat{F}_{r}^{l_{r}}$ be a factorization of $\widehat{f}$ into irreducible, non-associated and monic factors in $\mathcal{M}[y]$. Then there exists a canonical bijection between the factors $\widehat{F}_{1}, \ldots, \widehat{F}_{r}$ of the factorization of $\widehat{f}$ and the branches of $f$ at infinity. Moreover, the exponents of these factors are equal to the multiplicities of the corresponding branches.

Sketch of the proof. Since $P_{1}, \ldots, P_{k} \in U_{1}$, we put $Q_{i}=\varrho_{1}\left(P_{i}\right)$ and $g_{1}=f_{1} \circ \varrho_{1}^{-1}$. It suffices to show that there exists a canonical bijection between the factors $\widehat{F}_{1}, \ldots, \widehat{F}_{r}$ and the non-associated factors of the factorizations of the germs $\left(g_{1}\right)_{Q_{i}}, i=1, \ldots, k$, and that the exponents of the factors $\widehat{F}_{i}$ are equal to the exponents of the corresponding factors.

Let $\widehat{F}_{i}(y)=y^{n_{i}}+\widehat{\alpha}_{1}^{i} y^{n_{i}-1}+\ldots+\widehat{\alpha}_{n_{i}}^{i}, \widehat{\alpha}_{j}^{i} \in \mathcal{M}, n_{i} \geq 1, i=1, \ldots, r$. Take representatives $\alpha_{j}^{i}$ of the germs $\widehat{\alpha}_{j}^{i}$, defined in some $K(R), R>0$, such that $f=F_{1}^{l_{1}} \ldots F_{r}^{l_{r}}$ in $K(R) \times \mathbb{C}$ where $F_{i}(x, y)=y^{n_{i}}+\alpha_{1}^{i}(x) y^{n_{i}-1}+\ldots+$ $\alpha_{n_{i}}^{i}(x)$. Since there exists a canonical parametrization of the zero-set of $F_{i}$ in $K\left(R^{\prime}\right) \times \mathbb{C}$ for some $R^{\prime}>R$ (see [1]), we easily deduce that the closure of this set on the line $H_{\infty}$ is exactly one of the points $P_{j}$. Denote it by $P_{j(i)}$.

In the coordinates $(y, z)$ of the map $\varrho_{1}$ we have in the set $\{(y, z): 0<$ $|z|<1 / R\}, g_{1}(y, z)=f_{1} \circ \varrho_{1}^{-1}(y, z)=z^{n} f(1 / z, y / z)=G_{1}^{l_{1}}(y, z) \ldots G_{r}^{l_{r}}(y, z)$ where $G_{i}(y, z)=z^{n_{i}} F_{i}(1 / z, y / z)=y^{n_{i}}+z \alpha_{1}^{i}(1 / z) y^{n_{i}-1}+\ldots+z^{n_{i}} \alpha_{n_{i}}^{i}(1 / z)$. From (1) and the equality $f=F_{1}^{l_{1}} \ldots F_{r}^{l_{r}}$ it easily follows that each of the functions $z^{j} \alpha_{j}^{i}(1 / z)$ has a removable singularity at 0 . Hence each $G_{i}$ extends to a holomorphic function on $\{(y, z):|z|<1 / R\}$. From the definition of $G_{i}$ it easily follows that it vanishes only at one point on the line $z=0$, namely at $Q_{j(i)}$.

Next, one can easily check that the germ $\left(G_{i}\right)_{Q_{j(i)}}$ is irreducible and different from any other germ $\left(G_{l}\right)_{Q_{j(i)}}, l \neq i$. Thus the correspondence $\widehat{F}_{i} \mapsto\left(G_{i}\right)_{Q_{j(i)}}$ is the required bijection.

3. Analytic dependence of branches at infinity on a parameter. Let $f \in \mathbb{C}[x, y]$ have the form (1). Put $f^{\lambda}=f-\lambda, \lambda \in \mathbb{C}$. Denote by $D(\lambda, x)$ the discriminant of $f^{\lambda}$. By the definition, we have

(2) $\quad D(\lambda, x)= \pm R\left(f^{\lambda}, \partial f^{\lambda} / \partial y\right)$

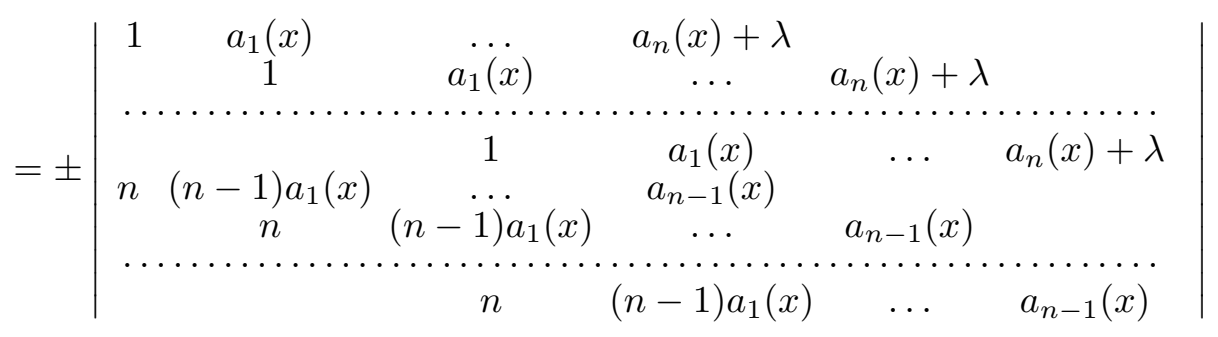


where $R\left(f^{\lambda}, \partial f^{\lambda} / \partial y\right)$ is the resultant of $f^{\lambda}$ and $\partial f^{\lambda} / \partial y$. From (2) we obtain $D(\lambda, x)=c_{0}(\lambda) x^{N}+c_{1}(\lambda) x^{N-1}+\ldots+c_{N}(\lambda)$ where $N \geq 0, c_{i} \in \mathbb{C}[\lambda]$. Obviously, $\operatorname{deg} c_{i} \leq n-1, i=0,1, \ldots, N$, and $c_{N}(\lambda)=( \pm 1) n^{n} \lambda^{n-1}+\ldots$ By the last equality, $D(\lambda, x)$ does not vanish identically. So, we can assume that $c_{0} \not \equiv 0$. Define $\Lambda(f)=\left\{\lambda \in \mathbb{C}: c_{0}(\lambda)=0\right\}$.

Lemma 1. The set $\Lambda(f)$ is finite and $\# \Lambda(f) \leq n-1$. Moreover, for each $\lambda_{0} \notin \Lambda(f)$, there exist a neighbourhood $U_{\lambda_{0}}$ of $\lambda_{0}$ and $R>0$ such that $D(\lambda, x) \neq 0$ for any $(\lambda, x) \in U_{\lambda_{0}} \times K(R)$.

Proof. The first assertion is obvious. Take $\lambda_{0} \notin \Lambda(f)$. By an elementary inequality for the zeros of a polynomial in one variable, all roots of $D\left(\lambda_{0}, x\right)=0$ lie in the closed disc centred at 0 and with radius $R^{\prime}=$ $2 \sup _{i}\left|c_{i}\left(\lambda_{0}\right) / c_{0}\left(\lambda_{0}\right)\right|^{1 / i}$. Take $R>R^{\prime}$. From the continuity of $c_{i}(\lambda) / c_{0}(\lambda)$, $i=1, \ldots, N$, at $\lambda_{0}$ it follows that there exists a neighbourhood $U_{\lambda_{0}}$ of $\lambda_{0}$ such that $2 \sup _{i}\left|c_{i}(\lambda) / c_{0}(\lambda)\right|^{1 / i}<R$ for $\lambda \in U_{\lambda_{0}}$. So, by the same elementary inequality, $D(\lambda, x) \neq 0$ for $(\lambda, x) \in U_{\lambda_{0}} \times K(R)$.

We shall now prove the main lemma on the analytic dependence of factors of the factorizations of $f^{\lambda}$ in $\mathcal{M}[y]$ on the parameter $\lambda$. The idea of the proof is taken from the local result given in [4].

Lemma 2. Let $f$ be a polynomial in two variables $x$ and $y$ whose coefficients are analytic functions of the parameter $\lambda, \lambda \in K\left(\lambda_{0}, \delta\right), \lambda_{0} \in \mathbb{C}$, $\delta>0$, and let $f$ have the form

$$
f(\lambda, x, y)=y^{n}+a_{1}(\lambda, x) y^{n-1}+\ldots+a_{n}(\lambda, x), \quad n \geq 1 .
$$

If there exists $R>0$ such that the discriminant $D(\lambda, x)$ of the polynomial (3) does not vanish at any point of $K\left(\lambda_{0}, \delta\right) \times K(R)$, then

(i) there exist $r, n_{1}, \ldots, n_{r} \in \mathbb{N}$ and monic polynomials $F_{i} \in \mathcal{O}\left(K\left(\lambda_{0}, \delta\right)\right.$ $\times K(R))[y]$ of degree $n_{i}, i=1, \ldots, r$, such that $n_{1}+\ldots+n_{r}=n$ and

$$
f=F_{1} \ldots F_{r} \quad \text { in } \mathcal{O}\left(K\left(\lambda_{0}, \delta\right) \times K(R)\right)[y]
$$

and, for any fixed $\lambda \in K\left(\lambda_{0}, \delta\right)$, the factors $F_{i}(\lambda, \cdot, \cdot)$ generate irreducible elements in $\mathcal{M}[y]$,

(ii) there exist $r$ holomorphic mappings $\Phi_{i}: K\left(\lambda_{0}, \delta\right) \times K\left(R^{1 / n_{i}}\right) \rightarrow$ $K(R) \times \mathbb{C}, i=1, \ldots, r$, of the form $\Phi_{i}(\lambda, t)=\left(t^{n_{i}}, \varphi_{i}(\lambda, t)\right)$, such that, for any fixed $\lambda \in K\left(\lambda_{0}, \delta\right)$ and $i \in\{1, \ldots, r\}$, the mapping $\Phi_{i}(\lambda, \cdot)$ parametrizes the zero-set of $F_{i}(\lambda, \cdot, \cdot)$ in $K(R) \times \mathbb{C}\left(\right.$ i.e. $\Phi_{i}(\lambda, \cdot)$ is a holomorphic bijection $)$ and $\varphi_{i}(\lambda, \cdot)$ is meromorphic at $\infty$.

Proof. Let $\theta \in \mathbb{R}$ be such that $R=\exp (-2 \pi \theta)$. Consider the polynomial $p(\lambda, w, y)=f(\lambda, \exp (2 \pi i w), y) \in \mathcal{O}(A)[y]$ where $A=\{(\lambda, w): \operatorname{Im} w<$ $\left.\theta, \lambda \in K\left(\lambda_{0}, \delta\right)\right\}$. The discriminant of $p$ is equal to $D(\lambda, \exp (2 \pi i w))$ and thus it vanishes nowhere in $A$. So, if $p(\lambda, w, y)=0$ for some $(\lambda, w) \in A$ and 
$y \in \mathbb{C}$, then $(\partial p / \partial y)(\lambda, w, y) \neq 0$. Since $p$ is a polynomial of degree $n$ and $A$ is a simply connected domain, therefore by the monodromy theorem there exist $n$ holomorphic functions $p_{1}, \ldots, p_{n}$ in $A$ with different values at each point $(\lambda, w) \in A$ such that

$$
p(\lambda, w, y)=\prod_{i=1}^{n}\left(y-p_{i}(\lambda, w)\right) \quad \text { in } \mathcal{O}(A)[y] .
$$

Note that $p(\lambda, w+1, y)=p(\lambda, w, y)$. Consequently, (5) implies that for each $i, 1 \leq i \leq n$, there exist $j, 1 \leq j \leq n$, such that $p_{i}(\lambda, w+1)=p_{j}(\lambda, w)$. Hence for each $i, 1 \leq i \leq n$, there exists $k(i), 1 \leq k(i) \leq n$, such that $p_{i}(\lambda, w+k(i))=p_{i}(\lambda, w)$. So, by a suitable renumbering of the $p_{i}$ we can divide the sequence $p_{1}, \ldots, p_{n}$ into cycles, i.e. there exist $r, n_{1}, \ldots, n_{r}$ such that $n_{1}+\ldots+n_{r}=n$ and the first $n_{1}$ functions form a cycle (which means that $p_{1}(\lambda, w+1)=p_{2}(\lambda, w), p_{2}(\lambda, w+1)=p_{3}(\lambda, w), \ldots, p_{n_{1}}(\lambda, w+1)=$ $\left.p_{1}(\lambda, w)\right)$, the next $n_{2}$ functions form a cycle, etc.

Consider the first cycle $p_{1}, \ldots, p_{n_{1}}$. Put $X=K\left(R^{1 / n_{1}}\right)$ and define $\varphi_{i}^{1}$ : $K\left(\lambda_{0}, \delta\right) \times X \rightarrow \mathbb{C}, i=1, \ldots, n_{1}$, by $\varphi_{i}^{1}(\lambda, t)=p_{i}\left(\lambda, n_{1} w\right)$, where $w=$ $(2 \pi i)^{-1} \log t$. Obviously, $\varphi_{i}^{1}$ is well-defined, holomorphic (because locally there exists a branch of $\log t$ in $X)$ and for any $(\lambda, t) \in K\left(\lambda_{0}, \delta\right) \times X$ the values $\varphi_{i}^{1}(\lambda, t), i=1, \ldots, n_{1}$, are different. Moreover, the functions $\varphi_{i}^{1}$, $i=1, \ldots, n_{1}$, form a Puiseux cycle, i.e. for each primitive $n_{1}$-root of unity $\varepsilon$ we can renumber the $\varphi_{i}^{1}$ in such a way that $\varphi_{i}^{1}(\lambda, t)=\varphi_{1}^{1}\left(\lambda, \varepsilon^{i-1} t\right)$ for each $i=1, \ldots, n_{1}$.

Note that for any fixed $(\lambda, t) \in K\left(\lambda_{0}, \delta\right) \times X$ the values $\varphi_{i}^{1}(\lambda, t), i=$ $1, \ldots, n_{1}$, are roots of the equation $f\left(\lambda, t^{n_{1}}, y\right)=0$ because for any $w$ such that $t=\exp (2 \pi i w)$ we have $f\left(\lambda, t^{n_{1}}, \varphi_{i}^{1}(\lambda, t)\right)=f\left(\lambda, \exp \left(2 \pi i n_{1} w\right)\right.$, $\left.\varphi_{i}^{1}(\lambda, \exp (2 \pi i w))\right)=p\left(\lambda, n_{1} w, p_{i}\left(\lambda, n_{1} w\right)\right)=0$. Hence

$$
f\left(\lambda, t^{n_{1}}, y\right)=\left(y-\varphi_{1}^{1}(\lambda, t)\right) \ldots\left(y-\varphi_{n_{1}}^{1}(\lambda, t)\right) \widetilde{f}(\lambda, t, y),
$$

where $\tilde{f} \in \mathcal{O}\left(K\left(\lambda_{0}, \delta\right) \times X\right)[y]$ is monic of degree $n-n_{1}$. Since the $\varphi_{i}^{1}$ form a Puiseux cycle, therefore $\prod_{i=1}^{n_{1}}\left(y-\varphi_{i}^{1}(\lambda, t)\right)=y^{n_{1}}+a_{1}^{1}\left(\lambda, t^{n_{1}}\right) y^{n_{1}-1}+$ $\ldots+a_{n_{1}}^{1}\left(\lambda, t^{n_{1}}\right)$, for some holomorphic $a_{j}^{i}$. Hence and from (6) we find that the coefficients of $\widetilde{f}$ also depend on $t^{n_{1}}$. So, putting $F_{1}(\lambda, x, y)=y^{n_{1}}+$ $a_{1}^{1}(\lambda, x) y^{n_{1}-1}+\ldots+a_{n_{1}}^{1}(\lambda, x)$ we have $f=F_{1} \widetilde{F}$ in $\mathcal{O}\left(K\left(\lambda_{0}, \delta\right) \times K(R)\right)[y]$, where $\widetilde{F}$ is monic of degree $n-n_{1}$.

Fix $\lambda \in K\left(\lambda_{0}, \delta\right)$. Since $\varphi_{i}^{1}(\lambda, \cdot) \in \mathcal{O}(X), i=1, \ldots, n_{1}$, satisfy the algebraic equation $f\left(\lambda, t^{n_{1}}, y\right)=0$, therefore they are meromorphic at $\infty$ (see Th. 14.2 in [5]). Hence the coefficients $a_{i}^{1}(\lambda, \cdot) \in \mathcal{O}(K(R)), i=1, \ldots, n_{1}$, of $F_{1}$ are also meromorphic at $\infty$. So, $F_{1}(\lambda, \cdot, \cdot)$ defines an element $\widehat{F}_{1}^{\lambda} \in \mathcal{M}[y]$. It is an irreducible element. In fact, otherwise we would have $\widehat{F}_{1}^{\lambda}=\widehat{G}_{1} \widehat{G}_{2}$ in $\mathcal{M}[y]$, where the $\widehat{G}_{i}$ are monic and $0<\operatorname{deg} \widehat{G}_{i}<n_{1}, i=1,2$. Taking 
representatives $G_{1}, G_{2}$ of $\widehat{G}_{1}, \widehat{G}_{2}$ with coefficients holomorphic in a $K(\widetilde{R})$, $\widetilde{R}>R$, such that $F_{1}(\lambda, \cdot, \cdot)=G_{1} G_{2}$ in $\mathcal{O}(K(\widetilde{R}))[y]$, we would easily check that for any $t \in K\left(\widetilde{R}^{1 / n_{1}}\right)$ the equation $G_{1}\left(t^{n_{1}}, y\right)=0$ has $n_{1}$ different roots $\varphi_{i}^{1}(\lambda, t), i=1, \ldots, n_{1}$, which is impossible.

For $(\lambda, t) \in K\left(\lambda_{0}, \delta\right) \times K\left(R^{1 / n_{1}}\right)$ we put $\varphi_{1}(\lambda, t)=\varphi_{1}^{1}(\lambda, t)$ and $\Phi_{1}(\lambda, t)=$ $\left(t^{n_{1}}, \varphi_{1}(\lambda, t)\right)$. From the above it follows that for any fixed $\lambda \in K\left(\lambda_{0}, \delta\right)$ the mapping $\Phi_{i}(\lambda, \cdot)$ parametrizes the zero-set of $F_{1}(\lambda, \cdot, \cdot)$ in $K(R) \times \mathbb{C}$.

Proceeding analogously with the remaining cycles in the sequence $p_{1}, \ldots, p_{n}$ we obtain $r$ polynomials $F_{1}, \ldots, F_{r} \in \mathcal{O}\left(K\left(\lambda_{0}, \delta\right) \times K(R)\right)[y]$ and the mappings $\Phi_{1}, \ldots, \Phi_{r}$, which satisfy (4) and the remaining assertions of the lemma.

Theorem 1. Let $f \in \mathbb{C}[x, y]$ be of the form (1). Then, for any $\lambda \notin \Lambda(f)$, each branch at infinity of the polynomial $f^{\lambda}=f-\lambda$ has multiplicity 1 and the number $r\left(f^{\lambda}\right)$ of branches at infinity is a constant independent of $\lambda$.

Pro of. Fix $\lambda_{0} \notin \Lambda(f)$. Then, by Lemma 1 , there exist $\delta>0$ and $R>0$ such that $D(\lambda, x)$ vanishes nowhere in $K\left(\lambda_{0}, \delta\right) \times K(R)$. Hence, from the first equality in (2) and a property of the resultant, and from the characterization of the branches at infinity given in our proposition, we obtain the first part of the theorem. Next, from Lemma 2 it follows that there exists $r \in \mathbb{N}$ such that, for any fixed $\lambda \in K\left(\lambda_{0}, \delta\right)$, the element $\widehat{f}^{\lambda} \in \mathcal{M}[y]$ generated by $f^{\lambda}$ is a product of $r$ irreducible factors in $\mathcal{M}[y]$. Hence, from the first part of the theorem and the proposition it follows that $r\left(f^{\lambda}\right)=r$ for $\lambda \in K\left(\lambda_{0}, \delta\right)$. So, the number of branches at infinity of $f^{\lambda}$ for $\lambda \in \mathbb{C} \backslash \Lambda(f)$ is locally constant, and hence constant, since $\mathbb{C} \backslash \Lambda(f)$ is connected.

From Lemma 2 it also follows that the branches at infinity of the polynomials of the pencil $f^{\lambda}, \lambda \notin \Lambda(f)$, and their parametrizations depend analytically on the parameter $\lambda$. Namely, we have

Theorem 2. Let $f \in \mathbb{C}[x, y]$ be of the form (1) and let $r$ be the constant number of branches at infinity of the polynomials $f^{\lambda}, \lambda \notin \Lambda(f)$. Then, for any $\lambda_{0} \notin \Lambda(f)$, there exist $\delta>0, R>0, n_{1}, \ldots, n_{r} \in \mathbb{N}$ and monic polynomials $F_{i} \in \mathcal{O}\left(K\left(\lambda_{0}, \delta\right) \times K(R)\right)[y]$ of degree $n_{i}$ such that $f^{\lambda}=F_{1} \ldots F_{r}$ in this ring and, for any fixed $\lambda \in K\left(\lambda_{0}, \delta\right)$, the polynomials $F_{i}(\lambda, \cdot, \cdot)$ generate irreducible non-associated elements in $\mathcal{M}[y]$. Moreover, there exist $r$ holomorphic mappings $\Phi_{i}: K\left(\lambda_{0}, \delta\right) \times K\left(R^{1 / n_{i}}\right) \rightarrow K(R) \times \mathbb{C}, i=1, \ldots, r$, of the form $\Phi_{i}(\lambda, t)=\left(t^{n_{i}}, \varphi_{i}(\lambda, t)\right)$ such that, for any fixed $\lambda \in K\left(\lambda_{0}, \delta\right)$ and $i \in\{1, \ldots, r\}$, the mapping $\Phi_{i}(\lambda, \cdot)$ parametrizes the zero-set of $F_{i}(\lambda, \cdot, \cdot)$ in $K(R) \times \mathbb{C}$, and $\varphi_{i}(\lambda, \cdot)$ is meromorphic at $\infty$.

Proof. By Lemma 1 , there exist $\delta>0, R>0$ such that $D(\lambda, x)$ vanishes nowhere in $K\left(\lambda_{0}, \delta\right) \times K(R)$. Hence, by Lemma 2, we obtain a 
factorization of $f^{\lambda}$ into factors $F_{i}$ and parametrizations $\Phi_{i}$ satisfying the assertion of the theorem.

3. Examples. In this section we shall give examples complementing the above considerations. The first example contradicts Engel's claim and shows that the numbers $r\left(f^{\lambda}\right)$ and $\widetilde{r}\left(f^{\lambda}\right)$ of branches at infinity are not lower semicontinuous. Moreover, it also shows that Abhyankar's statement, annouced by T. T. Moh (see Remark in [3]), is not true.

ExAmple 1. Put $f(x, y)=x y^{2}+y$. The points at infinity of each polynomial $f^{\lambda}, \lambda \in \mathbb{C}$, are $P_{1}=(1: 0: 0)$ and $P_{2}=(0: 1: 0)$. It is not hard to show that

$$
\begin{gathered}
r_{P_{1}}\left(f^{\lambda}\right)=\widetilde{r}_{P_{1}}\left(f^{\lambda}\right)= \begin{cases}1 & \text { for } \lambda \neq 0, \\
2 & \text { for } \lambda=0,\end{cases} \\
r_{P_{2}}\left(f^{\lambda}\right)=\widetilde{r}_{P_{2}}\left(f^{\lambda}\right)=1 \quad \text { for each } \lambda \in \mathbb{C} .
\end{gathered}
$$

Hence we obtain

$$
r\left(f^{\lambda}\right)=\widetilde{r}\left(f^{\lambda}\right)= \begin{cases}2 & \text { for } \lambda \neq 0 \\ 3 & \text { for } \lambda=0\end{cases}
$$

The second example, which was kindly indicated to me by Z. Jelonek, shows that $r\left(f^{\lambda}\right)$ and $\widetilde{r}\left(f^{\lambda}\right)$ are not, in general, upper semicontinuous.

ExAmple 2. Put $f(x, y)=y-(x y-1)^{2}$. The points at infinity of each polynomial $f^{\lambda}, \lambda \in \mathbb{C}$, are $P_{1}=(1: 0: 0)$ and $P_{2}=(0: 1: 0)$. It can be shown that

$$
\begin{gathered}
r_{P_{1}}\left(f^{\lambda}\right)=\widetilde{r}_{P_{1}}\left(f^{\lambda}\right)= \begin{cases}2 & \text { for } \lambda \neq 0, \\
1 & \text { for } \lambda=0,\end{cases} \\
r_{P_{2}}\left(f^{\lambda}\right)=\widetilde{r}_{P_{2}}\left(f^{\lambda}\right)=1 \quad \text { for each } \lambda \in \mathbb{C} .
\end{gathered}
$$

Hence we obtain

$$
r\left(f^{\lambda}\right)=\widetilde{r}\left(f^{\lambda}\right)= \begin{cases}3 & \text { for } \lambda \neq 0 \\ 2 & \text { for } \lambda=0 .\end{cases}
$$

Acknowledgement. I would like to thank to J. Chạdzyński for many helpful discussions.

\section{References}

[1] J. Chądzyński and T. Krasiński, Exponent of growth of polynomial mappings of $\mathbb{C}^{2}$ into $\mathbb{C}$, in: Singularities, S. Łojasiewicz (ed.), Banach Center Publ. 20, PWN, Warszawa 1988, 147-160.

[2] W. Engel, Ein Satz über ganze Cremona-Transformationen der Ebene, Math. Ann. 130 (1955), 11-19.

[3] T. T. Moh, On analytic irreducibility at $\infty$ of a pencil of curves, Proc. Amer. Math. Soc. 44 (1974), 22-24. 
[4] W. Pawłucki, Le théorème de Puiseux pour une application sous-analytique, Bull. Polish Acad. Sci. Math. 32 (1984), 555-560.

[5] S. Saks and A. Zyg mund, Analytic Functions, Monograf. Mat. 28, PWN, Warszawa 1965.

INSTITUTE OF MATHEMATICS

UNIVERSITY OF LÓDŹ

BANACHA 22

90-238 ŁÓDŹ, POLAND 